\title{
Effects of Ethnicity on the Relationship Between Vertical Jump and Maximal Power on a Cycle Ergometer
}

\author{
by \\ Majdi Rouis ${ }^{1,2}$, Laure Coudrat ${ }^{3}$, Hamdi Jaafar ${ }^{1}$, Elvis Attiogbé ${ }^{1}$, Henry Vandewalle ${ }^{4}$, \\ Tarak Driss ${ }^{1}$
}

The aim of this study was to verify the impact of ethnicity on the maximal power-vertical jump relationship. Thirty-one healthy males, sixteen Caucasian (age: $26.3 \pm 3.5$ years; body height: $179.1 \pm 5.5 \mathrm{~cm}$; body mass: $78.1 \pm 9.8$ $\mathrm{kg}$ ) and fifteen Afro-Caribbean (age: $24.4 \pm 2.6$ years; body height: $178.9 \pm 5.5 \mathrm{~cm}$; body mass: $77.1 \pm 10.3 \mathrm{~kg}$ ) completed three sessions during which vertical jump height and maximal power of lower limbs were measured. The results showed that the values of vertical jump height and maximal power were higher for Afro-Caribbean participants $(62.92 \pm 6.7 \mathrm{~cm}$ and $14.70 \pm 1.75 \mathrm{~W} \cdot \mathrm{kg}-1)$ than for Caucasian ones $(52.92 \pm 4.4 \mathrm{~cm}$ and $12.75 \pm 1.36 \mathrm{~W} \cdot \mathrm{kg}-1)$. Moreover, very high reliability indices were obtained on vertical jump (e.g. $0.95<$ ICC < 0.98) and maximal power performance (e.g. $0.75<$ ICC < 0.97). However, multiple linear regression analysis showed that, for a given value of maximal power, the AfroCaribbean participants jumped $8 \mathrm{~cm}$ higher than the Caucasians. Together, these results confirmed that ethnicity impacted the maximal power-vertical jump relationship over three sessions. In the current context of cultural diversity, the use of vertical jump performance as a predictor of muscular power should be considered with caution when dealing with populations of different ethnic origins.

Key words: power output, cycling, practice effect, reliability, ethnicity.

\section{Introduction}

Vertical jump tests are widely used as effective field evaluations of maximal power, a key indicator of the anaerobic metabolism. Indeed, several studies have demonstrated that maximal power of the lower limbs measured on a cycle ergometer (Pmax) is positively correlated with vertical jump height (VJ). This shows that the greater the maximal power of the lower limbs, the greater the vertical jump height (Driss et al., 1998; Vandewalle et al., 1987).

However, a number of studies have highlighted that the Pmax-VJ relationship is impacted by ethnicity (Rouis et al., 2015). For example, for a given value of VJ, Pmax of West African volleyball players was lower than Pmax of their Caucasian counterparts. Thus, the prediction of maximal power from vertical jump height appears to be biased when various ethnic groups are considered. This result is even more important today in the context of cultural diversity.

Earlier extensive studies in exercise physiology showed that ethnicity had an impact on power performance in jumping. All of these

\footnotetext{
1 - Laboratoire CeRSM (EA 2931), Equipe de Physiologie, Biomécanique et Imagerie du Mouvement, UFR STAPS, Université Paris Ouest Nanterre La Défense, Nanterre, France.

2 - Institut supérieur du sport et de l'éducation physique de Ksar-Sä̈, Manouba, Tunisie.

3 - LCOMS, EPSAP, Emotion-action, UFR SciFA, Département STAPS-Metz, Université de Lorraine, Metz, France.

4 - Laboratoire de Physiologie, UFR de Santé, Médecine et Biologie Humaine, Université Paris XIII, Bobigny, France.
} 
studies found that Afro-Carribean males (Rouis et al., 2015; Zajac et al., 2000), young boys (Babel et al., 2005; Ben Ayed et al., 2011; Ghesquière and Eeckels, 1984; Malina, 1988), and adolescents (Burfoot, 1992) jumped higher than their white counterparts. In addition, a greater ability of AfroCarribean participants has been extended more generally to locomotor skills in preschool children, for activities such as running, hopping, leaping or sliding (Goodway et al., 2010). These results have been explained mainly by constitutional differences between ethnic populations, such as differences in terms of body composition (Wagner and Heyward, 2007), muscle-tendon properties (McCarthy et al., 2006) and muscle fiber types (Bouchard et al., 1986).

However, little is known of the impact of ethnicity on cycling performance, with divergent results found in exercise physiology. While some studies showed that Pmax of Afro-Carribean participants was lower than that of their Caucasian counterparts (Ben Ayed et al., 2011; Rouis et al., 2015), others found that Pmax was similar in both ethnic groups (Burfoot, 1992) or higher for Caucasian compared with AfroCarribean participants (Zajac et al., 2000). Since cycling is a complex cyclical activity requiring a specific coordination of the lower limb muscles, few authors have suggested that the contrasting results obtained on Pmax performance might stem from a practice effect (Ben Ayed et al., 2011; Vandewalle et al., 1987). Nevertheless, this hypothesis has not yet been truly tested.

The present study served as a preliminary stage to validate this assumption. In an earlier reliability study, the effect of practice on power performance was observed beginning with the second session of a test (Hopkins et al., 2001). Therefore, it might be assumed that three sessions of a test should be sufficient to observe an improvement in Pmax and VJ for Afro-Caribbean and Caucasian participants, respectively. If a practice effect is observed, then the impact of ethnicity on the Pmax-VJ relationship will tend to disappear with an increase in the number of sessions. If no practice effect is observed, then the impact of ethnicity on the Pmax-VJ relationship will persist throughout the sessions and will reinforce the assumption that predicting maximal power from vertical jump height should be considered with caution when dealing with different ethnic groups.

\section{Material and Methods}

Participants

Thirty-one healthy and active men (sixteen Caucasian and fifteen Afro-Caribbean), involved in recreational sports activities four to five hours per week (jogging, soccer), volunteered for this study. Participants were matched for age, body height and mass (Table 1). None of the participants was a cyclist or a jumper. Moreover, $73 \%$ of the Caucasian participants, but only $27 \%$ of the Afro-Caribbean participants reported owning a bicycle. Each participant signed an informed consent form prior to the experimental procedures. The experimental protocol was approved by the Institutional Review Board of the Nanterre University. The study was conducted according to the guidelines of the Declaration of Helsinki.

\section{Procedures}

Since a practice effect in power tests can be observed beginning with the second session of a test (Hopkins et al., 2001), the participants of the present study completed three sessions (two days apart). Each session consisted of a vertical jump test followed by a force-velocity test, separated by a passive recovery period of 15 minutes.

\section{Vertical jump test}

A maximal countermovement vertical jump (VJ) was performed according to the procedure and the device described by Vandewalle et al. (1987). The VJ corresponded to the distance between the participant's body height and the level reached by his head at the peak of the jump. The participants performed the vertical jump using a countermovement of the trunk, legs and arms, but without a run. They performed two or three jumps with a 10 to $15 \mathrm{~s}$ recovery period between the trials. Thereafter, the participants were given $2 \mathrm{~min}$ to rest before jumping again until reaching their maximal performance. Approximately ten jumps were performed and the highest result was recorded for further analysis.

\section{Force-velocity test}

The force-velocity test was performed according to the protocol proposed by Vandewalle et al. (1987). This test consisted of repetitive short maximal sprints $(6 \mathrm{~s}$ each) against 
different braking forces. The test began with a breaking force equal to $19.6 \mathrm{~N}$. This braking force was increased by $19.6 \mathrm{~N}$ after 5 min of recovery. The same exercise was performed again until the participants were unable to reach a peak velocity greater than $100 \mathrm{rpm}$. The participants generally performed from 6 to 8 short all-out sprints. According to Vandewalle et al. (1987), maximal power of lower limbs $\left(\mathrm{P}_{\max }\right)$ was determined from the linear force-velocity relationship and was equal to:

$P_{\max }=0.5 \mathrm{~V}_{0} \times 0.5 \mathrm{~F}_{0}=0.25 \mathrm{~V}_{0} \mathrm{~F}_{0}$

where $V_{0}$ is assumed to be an estimate of maximal velocity at zero braking force and $\mathrm{F}_{0}$ is assumed to be the braking force corresponding to zero velocity (Vandewalle et al., 1987).

\section{Statistical analyses}

Analyses were performed using Sigma-stat software (Jandel, France). Data distribution normality was confirmed using the KolmogorovSmirnov distance test.

For each session, the effect of ethnicity on the $P_{\max }-\mathrm{VJ}$ relationship was studied by multiple linear regression analysis between the dependent variable $(\mathrm{VJ})$ and the independent variable $\left(\mathrm{P}_{\max }\right)$ plus a dummy variable $\mathrm{E}$ corresponding to ethnicity $(E=1$ for Afro-Caribbean and $E=0$ for Caucasian) according to the following model:

$$
\mathrm{VJ}=\mathrm{a}+\mathrm{bPmax}+\mathrm{cE}+\varepsilon
$$

where $\varepsilon$ is the error.

The effect of practice on the $\mathrm{VJ}$ and $\mathrm{P}_{\max }$ was analyzed using a two-way analysis of variance (ANOVA): session (session 1, session 2, session 3) and ethnic group (Caucasian, Afro-Caribbean), with repeated measures on the first factor.

Additionally, the practice effect was studied from reliability indices using the test-retest correlation coefficient (r), the standard error of estimation (SEE) and intraclass correlation coefficients (ICC) between each session.

For all analyses, the level of significance was set at $p<.05$.

\section{Results}

Table 2 presents summary data for all of the dependent variables.

\section{Results of multiple regressions}

For each session, multiple regression analysis revealed that the relationships between VJ, $P_{\max }$ and ethnicity were significant $(p<.001)$, with the correlation coefficient (r) ranging from
0.68 to 0.74 (Figure 1). The VJ values were also significantly correlated with both $P_{\max }$ and ethnicity $(p<.001)$.

Moreover, the multiple regression equations showed that, for the same $P_{\max }$, the Afro-Caribbean group jumped $8 \mathrm{~cm}$ higher on average than the Caucasian group, regardless of the session.

\section{Vertical jump performance}

The two-way ANOVA performed on vertical jump performance revealed a main effect of ethnicity $\left(\mathrm{F}_{1,29}=16,13, p<.001\right)$. The value of the vertical jump height of the Afro-Caribbean group $(62.92 \pm 6.7 \mathrm{~cm})$ was higher than that of the Caucasian group $(52.95 \pm 4.4 \mathrm{~cm})$. However, there was no session effect and no interaction between the session and ethnicity $(p>.05)$.

The results of the test-retest correlation coefficients and the SEE and ICC values showed high reliability for the vertical jump height for both groups (i.e. $r>0.94$, ICC $\geq 0.95$, $\mathrm{SEE} \leq 3.98 \%$ ) (Figure 2).

\section{Maximal power of lower limbs}

The two-way ANOVA showed only a significant main effect of ethnicity for $\mathrm{P}_{\max }\left(\mathrm{F}_{1,29}=\right.$ 13.04, $p<.001)$. The results indicated that the value of $P_{\max }$ for the Afro-Caribbean group (14.70 $\left.\pm 1.75 \mathrm{~W} \cdot \mathrm{kg}^{-1}\right)$ was greater than that of the Caucasian group $\left(12.75 \pm 1.36 \mathrm{~W} \cdot \mathrm{kg}^{-1}\right)$. However, no session effect and no interaction between the session and ethnicity were found $(p>.05)$.

All reliability indices showed high reliability for $P_{\max }$ for both groups (i.e. $r>0.80$, ICC $\geq 0.78$, SEE $\leq 6.32 \%$ ) (Figure 2 ).

\section{Discussion}

Based on previous studies (Rouis et al., 2015), the results of the present study indicated that ethnicity significantly impacted the Pmax-VJ relationship. Indeed, the results of the multiple linear regression analysis showed that, for a given value of Pmax, the Afro-Caribbean participants jumped on average $8 \mathrm{~cm}$ higher than the Caucasians. However, the results of the present study showed no practice effect. Indeed, the results of the ANOVA showed neither a session effect nor an interaction between the session and ethnicity in all dependent variables (i.e. Pmax and VJ). Furthermore, the reliability indices confirmed the results of the ANOVA. 

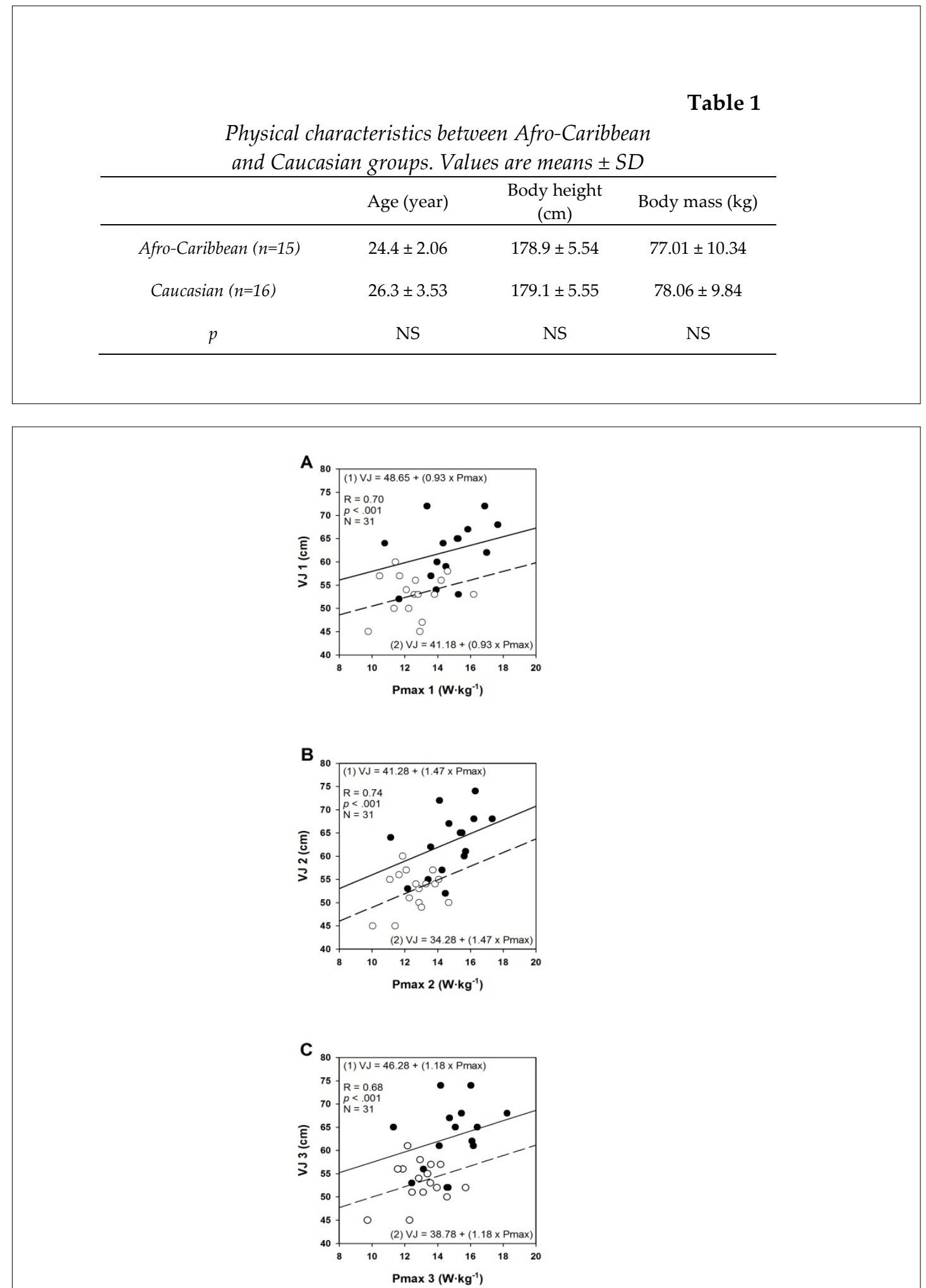

Figure 1

Relationships between maximal power ( $\left.P_{\max }\right)$ and vertical jump (VJ) for both ethnic groups. A) session 1, B) session 2 and $C$ ) session 3. Multiple regression equations for Afro-Caribbeans (1, continuous line, black circles) and Caucasians (2, dashed line, empty circles). 
Table 2

Trends of vertical jump performance $(V J)$ and maximal power $\left(P_{\max }\right)$ during the three sessions and according to the Caucasian and Afro-Caribbean groups. Values are means $\pm S D$

\begin{tabular}{llccc}
\hline & & Session 1 & Session 2 & Session 3 \\
\hline VJ & Caucasian & $52.92 \pm 4.52$ & $52.79 \pm 4.23$ & $53.14 \pm 4.38$ \\
$(\mathrm{~cm})$ & Afro-Caribbean & $62.33 \pm 6.41$ & $62.93 \pm 6.59$ & $62.80 \pm 7.12$ \\
& $p$ & $* * *$ & $* * *$ & $* * *$ \\
$\mathrm{P}_{\max }$ & Caucasian & $12.58 \pm 1.55$ & $12.61 \pm 1.18$ & $13.05 \pm 1.35$ \\
$\left(\mathrm{~W} \cdot \mathrm{kg}^{-1}\right)$ & Afro-Caribbean & $14.61 \pm 1.89$ & $14.66 \pm 1.63$ & $14.83 \pm 1.72$ \\
& $p$ & $* *$ & $* * *$ & $* *$ \\
\hline \multirow{2}{*}{$p:$ level of significance for the difference between } & groups (Caucasian versus Afro-
\end{tabular}
Caribbean); ${ }^{* *}: p<.001,{ }^{* *}: p<.01,{ }^{*}: p<.05$.
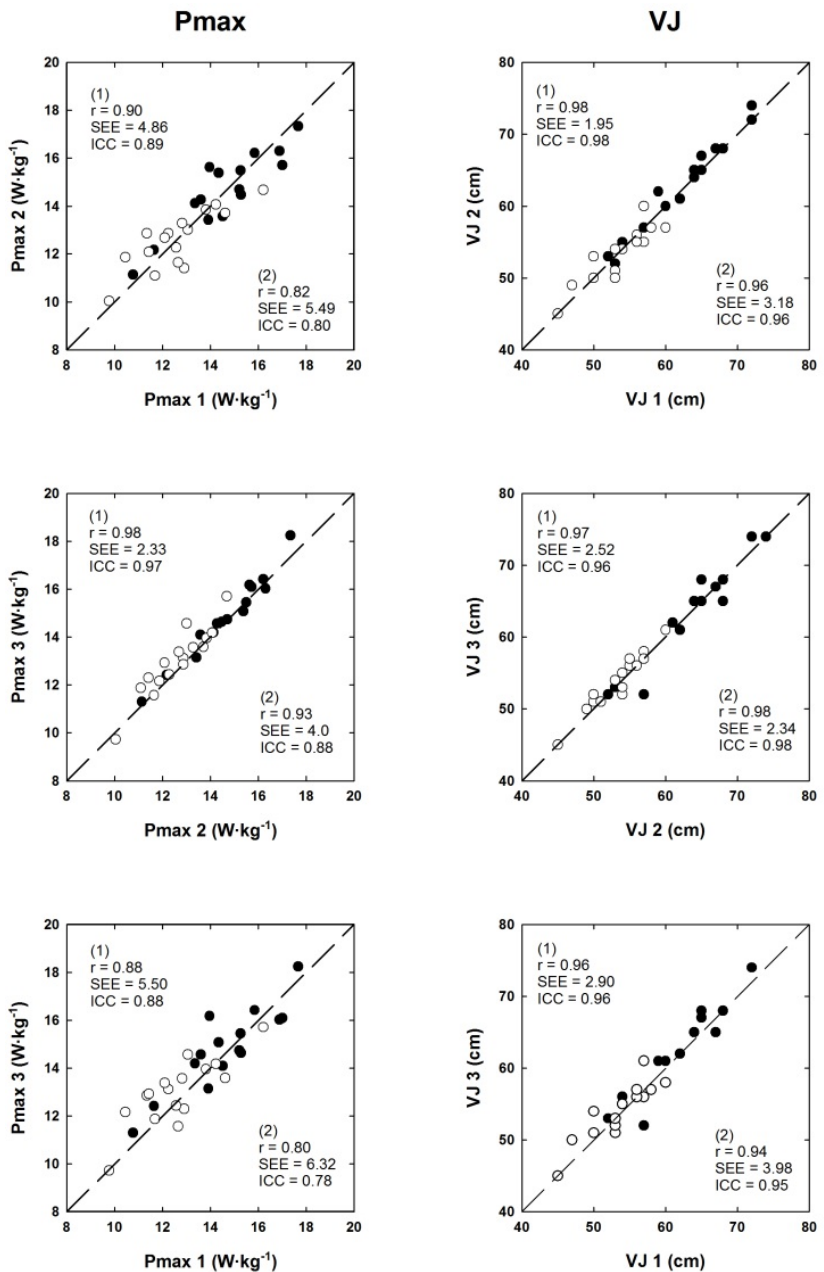

Figure 2

Reliability indices for maximal power $\left(P_{\max }\right)$ and vertical jump (VJ) between sessions in Afro-Caribbean (1, black circles) and Caucasian

(2, empty circles) groups. $r$ : test-retest correlation coefficient, SEE: standard error of estimation, ICC: intraclass correlation coefficient. The dashed line represents the identity line. 
Test-retest correlation coefficients, standard errors of estimation and intraclass correlation coefficients showed very high reliability between each session for both the Pmax and VJ performances and for both ethnic groups. Combined, these results demonstrated that neither the Caucasian nor Afro-Caribbean participants improved their performance in jumping and cycling, respectively.

Numerous studies in the field of physiology have highlighted morphological (Malina, 1988; Nelson et al., 1995), muscular (Ama et al., 1986), enzymatic (Saltin et al., 1995) and mechanical (Fukashiro et al., 2002) differences between Afro-Caribbean and Caucasian populations. Specifically, it has been demonstrated that Afro-Caribbean sedentary men and athletes have a higher percentage of fast twitch fibers and muscle stiffness than their Caucasian counterparts (Ama et al., 1986; Fukashiro et al., 2002). Furthermore, it has been demonstrated that vertical jump height and maximal power are positively correlated with the percentage of fast fibres (Glenmark et al., 1994) and muscle-tendon stiffness (Driss et al., 2012; Fukashiro et al., 2002).

Recently, some authors reported specific anatomical properties such as greater tendon length of the lower limbs in Afro-Caribbean subjects compared to Caucasian subjects (Kunimasa et al., 2014; Sano et al., 2013). Indeed, the greater tendon length may positively contribute to the output from the muscle tendon complex in a stretch shortening cycle during jumping with prestretch and this feature could be responsible for better vertical jump performance in the Afro-Caribbean participants.

Therefore, although the present study did not focus on these parameters, it could be assumed that better jumping and cycling performances observed in the Afro-Caribbean group compared with the Caucasian one might stem from these constitutional differences.

However, it is interesting to note that whereas all studies showed higher vertical jump performance for Afro-Caribbean participants compared with Caucasians (Ben Ayed et al., 2011; Burfoot, 1992; Zajac et al., 2000), divergent results were obtained for maximal power. Considering Pmax, the results of the present study are consistent with those obtained in the study of
Zajac et al. (2000). In this latter study, the AfroCaribbean group's performance in Pmax was $6.1 \%$ higher than that of the Caucasian group. In contrast, in the studies of Ben Ayed et al. (2011) and Rouis et al. (2015), Pmax of Afro-Caribbean participants was lower than that of their Caucasian counterparts. Moreover, the study of Burfoot (1992) found no ethnic difference in Pmax. These divergent results concerning the impact of ethnicity on Pmax might be interpreted as arising from a difference in the practice of cycling. However, the results obtained in the present study did not confirm this assumption as no effect of practice was observed over three sessions.

Moreover, a few studies from social science have demonstrated that cycling is an activity of white men (Steinbach et al., 2011) and that cycling barriers and opportunities varied according to ethnicity (Christie et al., 2011; Kidder, 2005). For example, young Africans in disadvantaged areas in England were more likely to report not owning or never having ridden a bicycle, compared with other ethnic groups such as whites (Christie et al., 2011). Thus, it is reasonable to postulate that the impact of ethnicity on the Pmax-VJ relationship might be explained in part by a socio-cultural factor, i.e. a practice effect.

A primary limitation of the present study relates to the number of sessions based on which a practice effect was measured. Indeed, it could be assumed that three sessions were not sufficient to detect a performance improvement in Pmax and/or the VJ. Further research is needed to understand the role of practice of cycling in the Pmax-VJ relationship on the basis of a larger-scale study integrating a greater number of sessions.

Moreover, the present study focused only on mechanical indices. It would be interesting to go further and focus on the inter-muscle coordination pattern, which is of great importance in cycling performance.

In conclusion, the results of the present study showed that, for a given value of Pmax, the Afro-Caribbean participants jumped higher than the Caucasians. This was the case regardless of the session. Furthermore, VJ and Pmax performances were similar from session 1 to session 3 and high reliability indices were found on these parameters between sessions and for 
both ethnic groups. Combined, these results suggested that the impact of ethnicity on the Pmax-VJ relationship was not influenced by a practice effect over three sessions. These findings call for caution when using vertical jump tests as an accuracy predictor of Pmax when different ethnic groups are considered. Further studies with more subjects and a longer practice period are required for more conclusive results.

\section{References}

Ama PF, Simoneau JA, Boulay MR, Serresse O, Theriaut G, Bouchard C. Skeletal muscle characteristics in sedentary black and Caucasians males. J Appl Physiol, 1986; 61: 1758-1761

Babel K, Hertogh C, Hue O. Influence of ethnic origin on predictive parameters of performance in sprint running in prepubertal boys. Int J Sports Med, 2005; 26: 1-5

Ben Ayed K, Latiri I, Doré E, Tabka, Z. Leg muscle power in 12-year-old black and white Tunisian football players. Res Sports Med, 2011; 19: 103-117

Burfoot A. White men can't run. Runner's World, 89-95; 1992

Bouchard C, Simoneau JA, Lortie G, Boulay MR, Marcotte M, Thibault MC. Genetic effects in human skeletal muscle fiber type distribution and enzyme activities. Can J Physiol Pharmacol, 1986; 64: 1245-1251

Christie N, Kimberlee R, Towner E, Rodgers S, Ward H, Sleney J, Lyons R. Children aged 9-14 living in disadvantaged areas in England: Opportunities and barriers for cycling. J Transport Geography, 2011; 19: 943-949

Driss T, Lambertz D, Rouis M, Vandewalle H. Influence of musculo-tendinous stiffness of the plantar ankle flexor muscles upon maximal power output on a cycle ergometer. Eur J Appl Physiol, 2012; 112: 3721-3728

Driss T, Vandewalle H, Monod H. Maximal power and force-velocity relationships during cycling and cranking exercises in volleyball players. Correlation with the vertical jump test. J Sports Med Phys Fitness, 1998; 38: 286-293

Fukashiro S, Abe T, Shibayama A, Brechue WF. Comparison of viscoelastic characteristics in triceps surae between black and white athletes. Acta Physiol Scand, 2002; 175: 183-187

Ghesquière J, Eeckels R. Health, physical development, and fitness of primary school children in Kinshasa. In J. Ilmarinen \& I. Välimäki (Eds.), Children and Sport. Paediatric work physiology. Berlin Heidelberg: Springer-Verlag, 1984; 20-30

Glenmark B, Hedberg G, Kaijser L, Jansson E. Muscle strength from adolescence to adulthood -relationship to muscle fibre types. Eur J Appl Physiol, 1994; 68: 9-19

Goodway JD, Robinson LE, Crowe H. Gender differences in fundamental motor skill development in disadvantaged pre-schoolers from two geographical regions. Res Q Exerc Sport, 2010; 81: 17-24

Hopkins WG, Schabort EJ, Hawley JA. Reliability of power in physical performance tests. Sports Med, 2001; 31: $211-234$

Kidder J. Style and action: a decoding of bike messenger symbols. J Contemp Ethnogr, 2005; 34: 344-367

Kunimasa Y, Sano K, Oda T, Nicol C, Komi PV, Locatelli E, Ito A, Ishikawa M. Specific muscle-tendon architecture in elite Kenyan distance runners. Scand J Med Sci Sports, 2014; 24: e269-274

Malina RM. Racial/ethnic variation in the motor development and performance of American children. Can J Appl Sport Sci, 1988; 13: 136-143

McCarthy JP, Hunter GR, Larson-Meyer DE, Bamman MM, Landers KA, Newcomer BR. Ethnic differences in triceps surae muscle-tendon complex and walking economy. J Strength Cond Res, 2006; 20: 511-8

Nelson DA, Jacobsen G, Barondess DA, Parfitt AM. Ethnic differences in regional bone density, hip axis length, and lifestyle variables among healthy black and white men. J Bone Miner Res, 1995; 10: 782-787

Rouis M, Attiogbé E, Vandewalle H, Jaafar H, Noakes TD, Driss T. Relationship between vertical jump and 
maximal power output of legs and arms: effects of ethnicity and sport. Scand J Med Sci Sports, 2015; 25: e197-207

Saltin B, Kim CK, Terrados N, Larsen H, Svedenhag J, Rolf CJ. Morphology, enzyme activities and buffer capacity in leg muscles of Kenyan and Scandinavian runners. Scand J Med Sci Sports, 1995; 5: 222-230

Sano K, Ishikawa M, Nobue A, Danno Y, Akiyama M, Oda T, Ito A, Hoffrén M, Nicol C, Locatelli E, Komi PV. Muscle-tendon interaction and EMG profiles of world class endurance runners during hopping. Eur J Appl Physiol, 2013; 113: 1395-403

Steinbach R, Green J, Datta J, Edwards P. Cycling and the city: A case study of how gendered, ethnic and class identities can shape healthy transport choices. Soc Sci Med, 2011; 72: 1123-1130

Vandewalle H, Pérès G, Heller J, Panel J, Monod H. Force-velocity relationship and maximal power on a cycle ergometer-correlation with a vertical jump. Eur J Appl Physiol, 1987; 56: 650-656

Wagner DR, Heyward VH. Measures of body composition in blacks and whites: a comparative review. Am J Clin Nutr, 2007; 71: 1392-1402

Zajac A, Waskiewicz Z, Litkowycz R. Motor fitness, aerobic and anaerobic power and physique in elite black and white athletes. J Hum Kinet, 2000; 4: 85-9

\section{Corresponding author:}

\section{Majdi Rouis}

Laboratoire CeRSM (EA 2931), Equipe de Physiologie, Biomécanique et Imagerie du Mouvement, UFR STAPS, Université Paris Ouest Nanterre La Défense, Nanterre, France.

Address: Université Paris Ouest Nanterre, UFR STAPS, bâtiment S, 200 avenue de la République, 92000 Nanterre cedex.

Phone number : +33-1-40975756

E-mail: majdi.rouis@laposte.net 